\title{
Singular Perturbation Analysis for Identification of Dynamic Behaviour and Stability of a Nonlinear Model of Long Term Progression of Diabetes Mellitus
}

\author{
CHONTITA RATANAKUL ${ }^{1,2}$, YONGWIMON LENBURY* ${ }^{1,2}$, JEERAWAN SUKSAMRAN $^{2,3}$ \\ ${ }^{1}$ Department of Mathematics, Faculty of Science, Mahidol University \\ Rama 6 rd., Bangkok 10400, THAILAND \\ ${ }^{2}$ Centre of Excellence in Mathematics, PERDO, THAILAND \\ ${ }^{3}$ Department of Mathematics, Faculty of Science \\ King Mongkut's University of Technology North Bangkok, Bangkok 10800, THAILAND
}

\begin{abstract}
There have been numerous attempts to model the progression of Diabetes Mellitus, which is a disease suffered by those with eating disorders with prevalence in the aged population. Models in the past have not been very successful in discovering the future development of the symptoms in a long term prediction. This is due to the fact that the state variables under consideration change in drastically different time scales, and the models that do not take careful account of this are not able to provide sufficiently accurate forecast that can be of satisfactory assistance to physicians taking care of their patients. In this work, we use the singular perturbation method to analyse a model of insulin and glucose interaction, incorporating beta cell dynamics and the pancreatic reserve, proposed by De Gaetano et al. in 2008. Different dynamic behaviour will be identified and numerical simulations will be carried out in support of our theoretical predictions.
\end{abstract}

Key-Words: - nonlinear model, stability, singular perturbation, Diabetes Mellitus, dynamic behavior

Received: March 9, 2020. Revised: September 2, 2020. Accepted: October 17, 2020. Published: November 2, 2020.

\section{Introduction}

Drug resistance has been a grave concern for many. According to [1], diabetes is on the rise across the globe. IDF's statistics shows that at the present time it is estimated that every seven seconds someone dies from diabetes or its complications, $50 \%$ of which deaths occurring to those under the age of 60 years, amounting to a total of 4 million deaths per year. The global diabetes prevalence is $8.8 \%(95 \%$ confidence interval $7.2-11.3 \%$ ) of the world population in 2017, standardized for the age group 20-79 years, according to [2].

In [3], the statistics indicates that, in 2017, Thailand has a population of 4.8 million who suffer from diabetes, only half of which has been diagnosed with the disease. In addition, it was reported in [4] that one in eleven people in the Thai population, at a mature age, is diagnosed with diabetes in 2016, while the World Health Organization reported that during 2009 - 2014, the number of diabetic patients has risen 4 folds and over 70 thousand died from illnesses related to diabetes each year [4].

Glycemia and insulinemia are regulated through a negative feedback loop in which $\beta$-cells are stimulated by plasma glucose to release insulin leading to insulin-mediated increased tissue glucose uptake and decreased liver gluconeogenesis and glycogenolysis [5].

In 2001, Lenbury et al. [6] proposed a nonlinear mathematical model of the glucose-insulin control mechanism, incorporating the function of beta-cells in maintaining and regulating plasma insulin level in human. A gastrointestinal absorption term is utilized to model glucose absorption by the intestine and the entry of glucose into the bloodstream, assuming that this process takes place at a given rate initially but declining exponentially with time. The model is analysed using the singular perturbation method by which the delineating conditions on the system parameters are derived to identify different dynamic behaviour, including the existence of limit cycles in the system model which mimic oscillatory patterns often observed in clinical data. A sinusoidal term is 
added to take into account the temporal absorption of glucose in order to investigate the patients' responses under ambulatory-fed conditions.

In 2015, Cao et al. [7] considered a model consisting of four states of type 2 diabetes, assuming no input or output. The model consists of partial differential equations for which a general well-posedness result is obtained and the exponential stability of dynamic solution is proved. The steady-state solution gives a prediction of the stable distribution probability of diabetics.

In 2008, Giang et al. [8] considered and analysed the delay model of the glucose-insulin system proposed by Palumbo et al. in [9], where persistence, as well as existence and stability of a unique positive equilibrium point have been proved. In this work [8], uniform persistence of such equilibrium solutions and their global stability are considered. The effect of delays is investigated in terms of oscillating solutions utilizing the omega limit set of a persistent solution and the full time solution which they were able to derive. The model is shown be globally stable and to admit slowly oscillating behaviour under suitable conditions on the system parameters.

In the same year, Kardar et al. [10] utilize an advisory/control algorithm to assist out-patients affected by insulin dependent diabetes mellitus. By using Mamdani type fuzzy logic controllers, their advisory/control algorithm puts together expert knowledge about the treatment of this diabetes in order to regulate the blood glucose level while the patient is subjected to disturbances in glucose levels due to food intakes or there are fluctuations in the measured glucose level arising from discrepancies in the measuring instrument

In 2009, Chuedoung et al. [11] proposed and analysed a delayed nonlinear model of glucoseinsulin system incorporating the beta cells as the third compartment. Later on, Lueabunchong et al. [12] carried out a statistical evaluation of a glucose/insulin nonlinear model of differential equations, using both classical and Bayesian procedures. Then, in 2013, Juagwon et al. [13] worked on the reconstruction of insulin secretion under the influences of hepatic extraction during OGTT.

Also in 2008, De Gaetano et al. [14] proposed a model of the pancreatic islet compensation, and proved some fundamental qualitative characteristics of its solutions. Its performance over the span of a lifetime is simulated under various conditions. Upon comparing between two previous models of diabetes progression, the proposed model is judged to be a realistic and robust description of the glucose- insulin process in healthy and diabetic person. Their model can be reduced to either "fast" or "slow" subsystems by varying the size of one of the system parameters.

Comparatively, the models mentioned above, while useful in answering several pertinent questions concerning Diabetes Mellitus progression, have not taken into account, in their models analyses, the extreme differences in the time scales at which the state variables vary with time. The model proposed by Cao et al. in 2015 [7\} is a system of partial differential equations, while those in [6], [8], [9], [11], [13] and [14] are systems of ordinary differential equations, incorporating different mechanisms deemed important into their models. These are deterministic models, whereas [12] carried out a statistical study of a glucose/insulin nonlinear model of interest.

Specifically, we observe that the model of De Gaetano et al. [14] has not incorporated appropriate expression to take into account the decline of the ability of the cells to absorb or use up blood sugar as the glucose level becomes higher. According to [15], in a person with prediabetes conditions, the pancreas has to work increasingly hard to release sufficient insulin to keep the blood glucose level down. Insulin resistance occurs when excess glucose in the blood reduces the ability of the cells to absorb and use blood sugar for energy. We therefore propose a modification of the model in [14] to incorporate the decline in the ability of cells in controlling plasma glucose when its level becomes higher. The singular perturbation technique is utilized to identify different dynamic behaviour permitted by our model.

\section{Model System}

The "original" model in [14] describes the time course of the glucose-insulin system of a single individual over many years, consisting of the following equations on the rates of change of $\beta$ cell mass $(B)$, fasting glucose concentration $(G)$, and fasting insulin concentration $(I)$ :

$$
\begin{aligned}
& \frac{d B}{d t}=\varepsilon \lambda B, B\left(t_{0}\right)=B_{0} \\
& \frac{d \eta}{d t}=\varepsilon\left[-K_{\eta G} G \eta+T_{\eta}\right], \eta\left(t_{0}\right)=\eta_{0} \\
& \frac{d G}{d t}=T_{g I}-K_{x g} G-K_{x g I} I G, G\left(t_{0}\right)=G_{0} \\
& \frac{d I}{d t}=h(G) T_{i g B} B-K_{x i} I, I\left(t_{0}\right)=I_{0}
\end{aligned}
$$

where 


$$
\begin{aligned}
& h(G)=\frac{\left(G / G_{h}\right)^{v_{h}}}{1+\left(G / G_{h}\right)^{v_{h}}}=\frac{G^{v_{h}}}{\alpha_{h}+G^{v_{h}}}, \alpha_{h}=G_{h}^{v_{h}} \\
& \lambda(G)=\lambda_{\min }+\eta \frac{x^{3}}{1+x^{3}}, x(G)=x_{0} \frac{G}{G_{\lambda}}
\end{aligned}
$$

while $\eta$ represents the capability of the pancreas to increase the reserved $\beta$-cells. The equation (1) for $B$ assumes a logistic growth rate that varies with the glucose level according to the expression (6). The first term on the right of (2) is the reduction in the capability of the pancreas due to increase in blood glucose, while $T_{\eta}$ is the spontaneous recovery rate of the pancreas. $T_{g I}$ is the rate of liver glucose output, $K_{x g}$ is the 1 st-order insulin-independent glucose tissue uptake rate, and the term $K_{x g I} I G$ in (3) is the 2nd-order insulin-dependent glucose tissue uptake rate per $\mathrm{pM}$ insulin, and represents insulin sensitivity. The first term in (4) is the rate of insulin release from the $\beta$-cells, while the last term is the apparent 1st-order elimination rate [14].

In order to reflect the previously mentioned symptom, observed in [15] that, in a person with prediabetes conditions, excess levels of glucose in the blood reduce the ability of the cells to absorb and use blood sugar for energy, we propose to replace equation (3) by the following equation:

$$
\frac{d G}{d \tau}=T_{g I}-K_{x g} G-K_{x g I} r(G) I G, G\left(t_{0}\right)=G_{0}
$$

where

$$
r(G)=\frac{G}{M+G^{2}}
$$

$M$ being a positive constant, so that the rate of removal of glucose, due to insulin, is no longer a constant multiple of the product of $G$ and $I$. It now increases with higher plasma glucose to a certain point, then declines with the increase of $G$ beyond a certain level, to more closely simulate the clinical condition mentioned in [15].

Moreover, since the model allows their levels to drop close to zero, it is considered more reasonable to take $G$ and $I$ in the model equations to be those concentrations above their respective basal levels.

The parameter $\varepsilon$ may be thought of as being a very small scaling factor. When $\varepsilon$ is allowed to be extremely small, we arrive at a "fast" model and the rates of variations of $B$ and $\eta$ over time also become very small, and therefore, $B$ and $\eta$ may be thought of as being slow in the fast time $t$, expressed, for example, in minutes. The "fast" subsystem becomes

$$
\begin{aligned}
& \frac{d B}{d t}=0, B\left(t_{0}\right)=B_{0} \Rightarrow B(t) \equiv B_{0} \\
& \frac{d \eta}{d t}=0, \eta\left(t_{0}\right)=\eta_{0} \Rightarrow \eta(t) \equiv \eta_{0} \\
& \frac{d G}{d \tau}=T_{g I}-K_{x g} G-K_{x g I} r(G) I G, G\left(t_{0}\right)=G_{0} \\
& \frac{d I}{d t}=h(G) T_{i g B} B-K_{x i} I, I\left(t_{0}\right)=I_{0}
\end{aligned}
$$

A large $\varepsilon$ yields a "slow" model which focuses on changes over months or years [14], providing long-term forecasts. In such a situation, it is proposed that we use of the following "slow" subsystem.

$$
\begin{aligned}
& \frac{d B}{d t}=\lambda B, B\left(t_{0}\right)=B_{0} \\
& \frac{d \eta}{d t}=-K_{\eta G} G \eta+T_{\eta}, \eta\left(t_{0}\right)=\eta_{0} \\
& G=\frac{\gamma}{\rho+r I}, \rho=\frac{K_{x g}}{K_{x g I}} \\
& I=h(G) I_{\max B} B
\end{aligned}
$$

We consider here in the "fast" model (9) - (12), with (5) - (6). In this case, compared to changes in $G$ and $I$, those in $B$ may be thought of as being quite slow so that $B$ may be thought of as remain relatively constant in time at $B_{0}$.

De Gaetano et al. [14] also carried out a detailed discussion of the numerical values assigned to their model system parameters. Guided by these values, we may observe that the parameters in the rate equation for $I$ are significantly smaller than those in the rate equation for $G$. Thus, to apply the singular perturbation analysis, it is reasonable to assume that $G$ is the faster variable, $I$ is the intermediate variable, and $\eta$ is the slow variable. We therefore modify Equations (9)-(10) by substituting $K_{\eta G}=\delta K_{\eta G}^{\prime}, T_{\eta}=\delta T_{\eta}^{\prime}, T_{i g B}=\delta T_{i g B}^{\prime}, K_{x i}=\delta K_{x i}^{\prime}$ and then dropping the primes, so that our model of interest consists of the following equations.

$$
\begin{aligned}
& \frac{d B}{d t}=0, B\left(t_{0}\right)=B_{0} \Rightarrow B(t) \equiv B_{0} \\
& \frac{d \eta}{d \tau}=\varepsilon \delta\left[-K_{\eta G} G \eta+T_{\eta}\right], \eta\left(t_{0}\right)=\eta_{0} \\
& \frac{d G}{d \tau}=T_{g I}-K_{x g} G-K_{x g I} r(G) I G, G\left(t_{0}\right)=G_{0}
\end{aligned}
$$


$\frac{d I}{d \tau}=\delta\left[h(G) T_{i g B} B-K_{x i} I\right], I\left(t_{0}\right)=I_{0}$

where $h(G)$ and $r(G)$ are as defined in (5) and (8), respectively.

Thus, when $\varepsilon$ and $\delta$ are very small, we may at first take $\eta$ to be relatively constant. $I$ changes at an intermediate speed, and $G$ moves at a relatively high speed.

\section{Singular Perturbation Analysis}

We begin by identifying the equilibrium manifolds in the $(G, I, \eta)$ - space.

\section{Slow Manifold $\dot{\eta}=0$}

This is the surface in the 3 dimensional space derived by equating the right hand side of (18) to zero, which yields

$$
G=\frac{T_{\eta}}{K_{\eta G} \eta}
$$

\section{Intermediate Manifold $\dot{I}=0$}

This is the surface in the 3 dimensional space derived by equating the right hand side of (19) to zero, which yields

$$
I=\frac{h(G) T_{i g B}}{K_{x i}}=\frac{\omega G^{v_{h}}}{\alpha_{h}+G^{v_{h}}}, \omega=\frac{T_{i g B} B_{0}}{K_{x i}}
$$

We observe that, on this manifold, we have $I=0$ when $G=0$, and $I \rightarrow \omega$ as $G \rightarrow \infty$

\section{Fast Manifold $\dot{G}=0$}

This is the surface in the 3 dimensional space derived by equating the right hand side of (20) to zero, which yields

$$
I=\frac{T_{g I}-K_{x g} G}{K_{x g I} r(G)}=\frac{\left(T_{g I}-K_{x g} G\right)\left(M+G^{2}\right)}{K_{x g I} G}
$$

We observe that on this manifold, $I \rightarrow \infty$ as $G \rightarrow 0$, $I \rightarrow-\infty$ as $G \rightarrow \infty$ and $I=0$ at

$$
G=\frac{T_{g I}}{K_{x g}}=G^{*} .
$$

\section{Letting}

$$
b=-\frac{T_{g I}}{2 K_{x g}}, c=0, d=\frac{T_{g I} M}{2 K_{x g}}
$$

we can prove the following result.

Lemma 1 The curve resulting from the intersection between the fast manifold $\dot{G}=0$ and the slow manifold $\dot{\eta}=0$ has 2 relative extrema at $G=G_{M 1}>0$ and $G=G_{M 2}>G_{M 1}>0$ provided

$$
d<-\frac{4}{27} b^{3}
$$

Proof

Differentiating (23) with respect to $I$, we obtain

$\frac{d I}{d G}=\frac{T_{g I} G^{2}-2 K_{x g} G^{3}-T_{g I} M}{K_{x g I} G^{2}}$

Thus, the relative extrema occur if the numerator of (26) vanishes, that is

$$
G^{3}-\frac{T_{g I}}{2 K_{x g}} G^{2}+\frac{T_{g I} M}{2 K_{x g}}=0
$$

The discriminant of (27) is

$$
\Delta=-4 b^{3} d-27 d^{2}
$$

which is positive since (25) holds. Hence, by the Descartes' Rule of Signs, we conclude that (27) has 2 positive solutions, which means there are 2 relative extrema on this curve with $G_{M 1,2}$ positive.

Let the two relative extrema on the fast manifold be denoted by $\left(G_{M 1}, I_{M 1}\right)$ and $\left(G_{M 2}, I_{M 2}\right)$ where

$$
I_{M 1,2}=\frac{T_{g I}-K_{x g} G_{M 1,2}}{K_{x g I} r\left(G_{M 1,2}\right)}
$$

We note here that

$G_{M 1,2}^{3}-\frac{T_{g I}}{2 K_{x g}} G_{M 1,2}^{2}+\frac{T_{g I} M}{2 K_{x g}}=0$

Thus,

$T_{g I}-K_{x g} G_{M 1,2}=\frac{T_{g I} M+K_{x g} G_{M 1,2}^{3}}{G_{M 1,2}^{2}}>0$

By (28), we see that $I_{M 1,2}>0$, which means the relative extrema are in the first quadrant of the $(G, I)$ plane.

The graphs of the intermediate and fast manifolds projected onto the $(G, I)$-plane may be visualized as shown in Figure 1, provided the conditions in the following theorem hold, where

$$
I_{1,2}=\frac{h\left(G_{M 1,2}\right) T_{i g B}}{K_{x i}}
$$

are the values of $I$ on the intermediate manifold $\dot{I}=0$ at which $G=G_{M 1}$ and $G_{M 2}$.

Theorem 1 The model system (17)-(20) admits a periodic solution in the form of a limit cycle surrounding the equilibrium point $\left(G_{c}, I_{c}, \eta_{c}\right)$, at which the 3 manifolds intersect, if (25) holds, and

Proof

$$
I_{1}<I_{M 1}, I_{M 2}<I_{2}
$$


If (29) holds, then the fast and intermediate equilibrium manifolds, projected onto the $(G, I)$ plane, will be located as shown in Figure 1 relative to each other.

Starting from a general initial point $\mathrm{P}\left(G_{0}, I_{0}, \eta_{0}\right)$ seen in Figure $1, \eta$ moves at a very slow speed to end up eventually at the slow manifold given by (21), not shown in Figure 1 . Meanwhile, $G$ and $I$ will vary as described below at rates which do not depend on $\eta$.

$G$ is the fast variable, so it will make a fast transition in the direction of increasing $G$ since here $\dot{G}>0$ in this region below the fast manifold. Here, a fast transition is denoted by a double arrow in Figure 1, while the intermediate one is denoted by a single arrow.

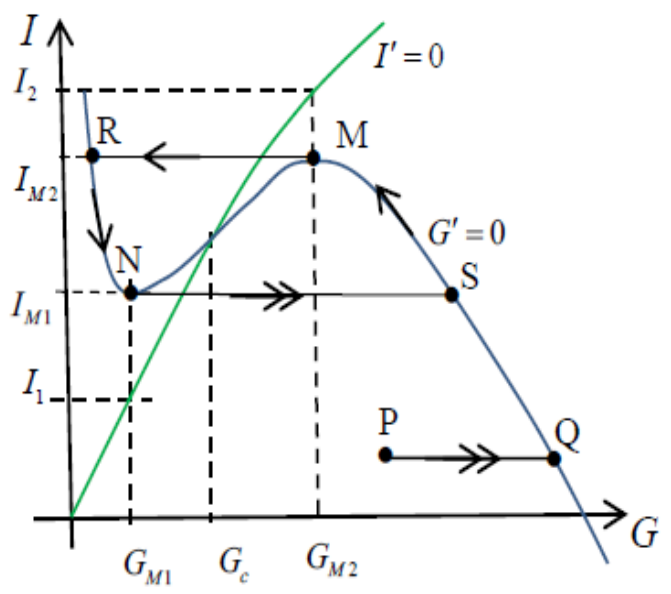

Figure 1. The fast and intermediate manifolds are here projected onto the $(G, I)$ - plane with transitions of fast speed (double arrows) and intermediate speed (single arrow)

Once the transition reaches the fast manifold at the point $\mathrm{Q}$, where $\dot{G}=0$ so that the transition will remain on this curve. Since on this side of the intermediate manifold $\dot{I}>0$, a transition at intermediate speed will be made upward along this curve until the maximum point $\mathrm{M}\left(G_{M 2}, I_{M 2}\right)$ is reached. The stability of the manifold is lost here so that a fast transition in the direction of decreasing $G$ will be made until we arrive at the fast manifold at the point R. Here, $\dot{I}<0$ so that a transition is made downward along this curve until the minimum point $\mathrm{N}\left(G_{M 1}, I_{M 1}\right)$ where the stability is again lost. A fast transition results which takes the trajectory towards the right branch of the fast manifold at the point $\mathrm{S}$, thereby closing the cycle and the trajectory keeps repeating around the same path SMRNS resulting in a limit cycle solution to the model system.

By a similar argument, we can show the following stability result.

Theorem 2 The model system (12)-(14) admits an equilibrium solution, $\left(G_{c}, I_{c}, \eta_{c}\right)$, which is locally asymptotically stable provided (21) holds, and either 1) $I_{1}>I_{M 1}$, and $I_{2}>I_{M 2}$, or

2) $I_{1}<I_{M 1}$, and $I_{2}<I_{M 2}$.

The equilibrium point will be a spiral node, and the trajectory will converge towards the stable steady state $\left(G_{c}, I_{c}, \eta_{c}\right)$.

The above two cases, in Theorem 1 and 2, describe a condition in which the insulin-mediated glucose elimination process is still functioning relatively well.

However, if (25) is violated, situations may occur in which the solution trajectory spirals away from the steady equilibrium state, thus leading to an underirable scenario where excess glucose can no longer be kept at a reasonable level, unless treatment intervention is prescibed appropriately. This then depicts the patterns observed in a person with prediabetes conditions or full onset of Diabetes Mellitus.

\section{Discussion}

We have analyzed a model of glucose-insulin control mechanism which incorporates the compartments of beta cell mass and the capacity of the pancreas in producing beta cells on reserve. The model is investigated in terms of the existence of limit cycle behavior which reflects the oscillatory dynamics frequently observed in experimental data.

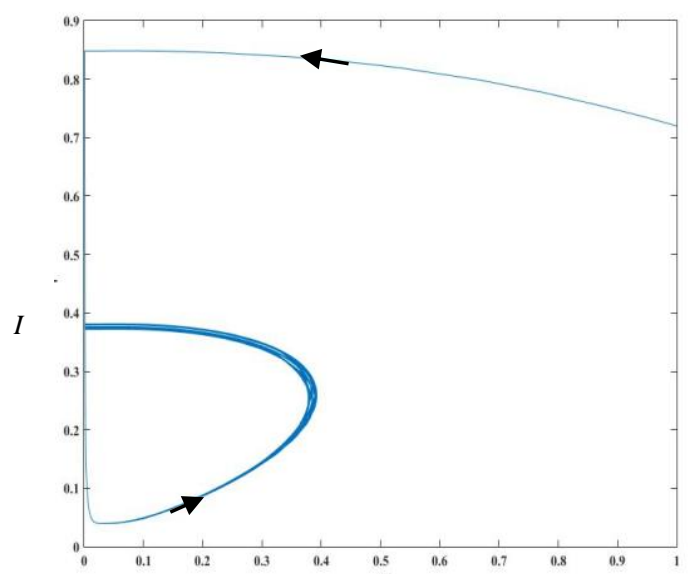

G 
Figure 2. Numerical solution of (12) - (14) projected onto the $(G, I)$ - plane, in the case that the conditions in Theorem 1 are satisfied. The trajectory tends toward a limit cycle as predicted by the theorem.

We show in Figure 2 the solution trajectory, projected onto the $(G, I)$ - plane, in the case where the conditions in Theorem 1 hold. We see that the trajectory traces out a limit cycle exactly as described by our singular perturbation arguments.

Starting at a point where glucose level is low, if the level begins to increase, we see that there is a small lag time in insulin response during which insulin level still decreases so that there is a delay in the response of the cells to the rise in glucose level. This is a reasonable occurrence since a response should be elicited only when the glucose level becomes too high and not before that.

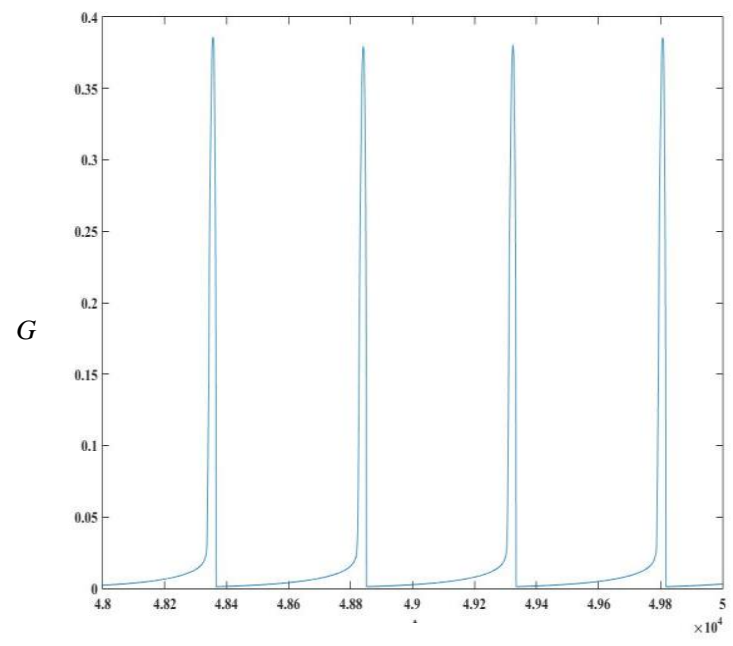

a)

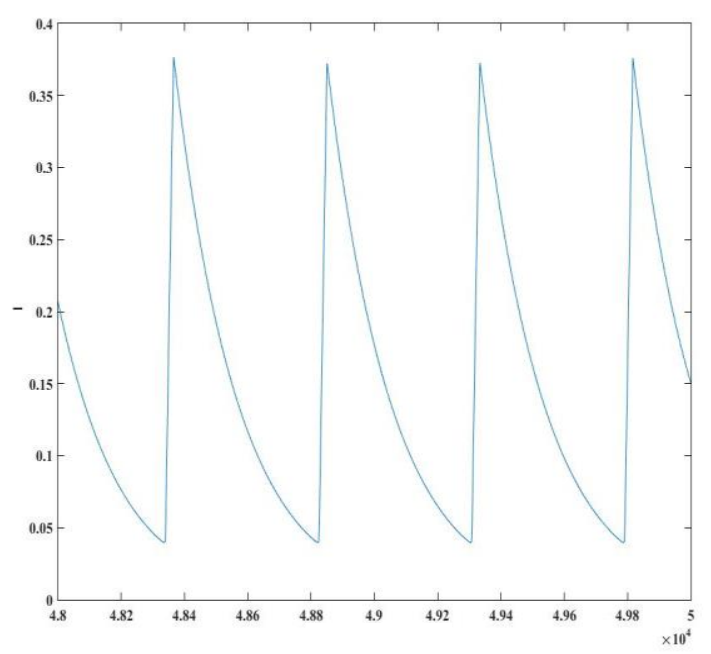

Figure 3. Times series of the solution of (12) - (14) seen in Figure 2 shows periodic behavior in a) glucose concentration, and b) insulin concentration.

When the glucose level has begun to drop in Figure 2, insulin level still increases and remains high until the glucose level reduces down to an extremely low level before insulin level drops quickly, as commonly observed in reported experimental data, for example, in [16].

Figure 3 shows the corresponding time series of glucose and insulin levels in the case where the 2 variables exhibit sustained oscillations.

In Figures 2 and $3, G_{0}=5 ; I_{0}=0.05 ; \eta_{0}=0.04$; $M=0.0005 ; B_{0}=1000 M ; T_{i g B}=0.4592 ; T_{g l}=$ $0.0738 ; K_{x g l}=0.08 ; K_{x g}=0.054 ; T_{\eta}=0.0016 ; K_{x i}=$ $0.08 ; K_{n G}=0.8 ; G_{h}=0.09, \varepsilon=0.25, \delta=0.25$. We found that $G_{M 1}=0.02274, G_{M 2}=0.68260, I_{1}=$ $0.02331, I_{2}=5.73827, I_{M 1}=0.04057, I_{M 2}=0.31553$, rounded to 5 decimal places. The parametric values assignment has been guided by De Gaetano's set of values to the orders of magnitude. Adjustments of values have been made in case where the conditions required by the theorem need to be satisfied.

\section{Conclusion}

In trying to efficiently control blood glucose levels, a great deal of complexities is involved in the treatment of symptoms. The use of traditional algorithm that is "one-size-fits-all" may not be flexible enough and rarely considers important factors that play crucial roles in the diabetic patients [17]. Many articles have stressed how mathematical models are playing important roles in the personalized treatment and management of Diabetes Mellitus and personalized medicine in general [1725]. A great deal of data is now readily available for model construction and verification. Sophisticated models can be a viable tool and should be placed at the disposal of physicians to assist them in their decision making concerning dosage prescriptions and changing treatment protocols which can be tailored to each patient in a personalized fashion.

\section{Acknowledgement}

Appreciation is extended toward Mahidol University, and the Centre of Excellence in Mathematics, CHE, Thailand, for financial support.

References: 
[1] International Diabetes Federation. IDF Diabetes Atlas, 8th edition. Brussels, Belgium: International Diabetes Federation, 2017. http://www.diabetesatlas.org; last accessed on January 23, 2019.

[2] E. Standl. Global Statistics on Diabetes, Forschergruppe Diabetes eV, Munich Helmholtz Centre, Germany for the EAPC Diabetes and CVD Educational Programme, European Society of Cardiology, https://www.escardio.org/Education/ Diabetes-and-CVD/Recommended-Reading/globalstatistics-on-diabetes.

[3] C. Deerochanawong. We Need to Unite to Change Diabetes. https://www.novonordisk.com/ content/dam/Denmark/HQ/sustainablebusiness/perf ormance-on-tbl/more-about-how-we-work/Creating $\% 20$ shared $\% 20$ value/PDF/Thailand $\% 20$ Blueprint $\%$ 20for\%20Change_2017.pdf.

[4] C. Nokdee, (2016) Diabetes is on the Rise. https://www.thaihealth.or.th/ Content/33953.

[5] A. De Gaetano, T. Hardy, B. Beck, E. AbuRaddad, P. Palumbo, J. Bue-Valleskey, N. Pørksen, Mathematical Models of Diabetes Progression, Am. J. Physiol. Endocrinol. Metab., Vol.295, No.6, 2008, pp. E1462-E1479. First published September 9, 2008; doi:10.1152/ajpendo.90444. 2008.

[6] Y. Lenbury, S. Ruktamatakul, S. Amornsamarnkul, Modeling Insulin Kinetics: Responses to a Single Oral Glucose Administration or Ambulatory-fed Conditions, Bio Systems, 2001. DOI:10.1016/S0303-2647(00) 00136-2.

[7] X. Cao, D. Liu, Suzhi, Y. Wang, Mathematical Modelling and Stability Analysis for Diabetes Predicting System, WSEAS Transaction on Mathematics, Vol.14, 2015, pp. 178-191.

[8] D. V. Giang, Y. Lenbury', A. De Gaetano, Delay Model of Glucose-Insulin Systems: Global Stability and Oscillated Solutions Conditional on Delays, Journal of Mathematical Analysis and Applications, Vol. 343, Issue 2, 2008, pp. 996-1006.

[9] P. Palumbo, S. Panunzi, A. De Gaetano, Qualitative Behavior of a Family of DelayDifferential Models of the Glucose-Insulin System, Discrete Contin. Dyn. Syst. Ser. B, Vol.2007, No.7, 2007, pp. 399-424.

[10] L. Kardar, A. Fallah, S. Gharibzadeh, F. Moztarzadeh, Application of Fuzzy Logic Controller for Intensive Insulin Therapy in Type 1 Diabetic Mellitus Patients by Subcutaneous Route, WSEAS Transactions on Systems and Control, Vol. 3, No. 9, 2008.

[11] M. Chuedoung, W. Sarika, Y. Lenbury, Dynamical Analysis of a Nonlinear Model for Glucose-Insulin System Incorporating Delays and bCells Compartment. Journal of Nonlinear Analysis
Series A: Theory and Methods, Vol. 71, No.12, 2009, pp. e1048-e1058.

[12] S. Lueabunchong, Y. Lenbury, S. Panunzi, A. Matone, Statistical Evaluation of a Glucose/Insulin Nonlinear Differential Equation Model with Classical and Bayesian Procedures. Recent Researches in Applied Computers and Computational Science, 2012, pp. 35-40.

[13] K. Juagwon, Y. Lenbury, A. De Gaetano, P. Palumbo, Reconstruction of Insulin Secretion under the Effects of Hepatic Extraction during OGTT: A Modelling and Convolution Approach, Recent Advances in Mathematics, WSEAS Proceedings, 2013, pp. 85-90.

[14] A. De Gaetano, T. Hardy, B. Beck, E. AbuRaddad, P. Palumbo, J. Bue-Valleskey, N. Pørksen, Mathematical Models of Diabetes Progression, Am. J. Physiol. Endocrinol. Metab., Vol.2008, No.295, pp.1462-1479.

[15] A. Felman, What to Know about Insulin Resistance. MedicalNewsToday, medically reviewed by Deborah Weatherspoon on March 26, 2019.

https://www.medicalnewstoday.com/articles/305567 .php

[16] O. Tschritter, A. Fritsche, F. Shirkavand, F. Machicao, H. H. Aring, M. Stumvoli, Assessing the Shape of the Glucose Curve During an Oral Glucose Tolerance Test, Diabetes Care, Vol. 26, 2003, pp. 1026-1033.

[17] S. Subramanian, I. B. Hirsch. Personalized Diabetes Management: Moving from Algorithmic to Individualized Therapy, Diabetes Spectr, Vol.27, No.2, 2014, pp. 87-91.

[18] D. C. Klonoff, Precision Medicine for Managing Diabetes, PubMed, December 29, 2014, https://doi.org/10.1177/1932296814563643.

[19] S. Del Prato, J. LaSalle, S. Matthaei, C. Bailey, Tailoring Treatment to the Individual in Type 2 Diabetes Practical Guidance from the Global Partnership for Effective Diabetes Management, International Journal of Clinical Practice, Vol.64, No.3, 2010, pp. 295-304, DOI: 10.1111/j.17421241.2009.02227.x. PubMed.

[20] B. P. Kovatchev, Diabetes Technology: Markers, Monitoring, Assessment, and Control of Blood Glucose Fluctuations in Diabetes, Scientifica (Cairo), Vol.2012, 2012, 283821, published online 2012 Oct 17. doi: $10.6064 / 2012 / 283821$

[21] G. Zhao, D. Wirth, I. Schmitz, M. MeyerHermann, A Mathematical Model of the Impact of Insulin Secretion Dynamics on Selective Hepatic Insulin Resistance. Nature Communications, Vol. 8, No.1362, 2017. 
[22] D. M. Eddy, L. Schlessinger, A Trial-Validated Model of Diabetes. Archimedes: Diabetes Care, Vol.26, No.11, 2003, pp. 3093-3101. https://doi.org/ 10.2337/diacare.26.11.3093

[23] E. K. Lee, X. Wei, F. Baker-Witt, M. D. Wright, A. Quarshie, Outcome-Driven Personalized Treatment Design for Managing Diabetes, INFORMS Journal on Applied Analytics, Vol.48, No.5, 2018. https://doi.org/10.1287/ inte.2018.0964. [24] G. C. Castellani, G. Menichetti, P. Garagnani, M. G. Bacalini, C. Pirazzini, C. Franceschi, S. Collino, C. Sala, D. Remondini, E. Giampieri, E. Mosca, M. Bersanelli, S. Vitali, I. F. Valle, P. Liò, L. Milanesi, Systems Medicine of Inflammaging. Briefings in Bioinformatics, Vol.17, No.3, 2015, pp. 527-540, DOI: 10.1093/bib/bbv062.

[25] P. Borovska, Big Data Analytics and Internet of Medical Things Make Precision Medicine a Reality. 18th International Conference on Applied Computer and Applied Computational Science (ACACOS '18), WSEAS Conference, Paris, France, April 13-15, 2018.

\section{Creative Commons Attribution License 4.0 (Attribution 4.0 International, CC BY 4.0)}

This article is published under the terms of the Creative Commons Attribution License 4.0

https://creativecommons.org/licenses/by/4.0/deed.en_US 